\title{
Effect of Germination Initiation on Competitive Capacity of Trichoderma atroviride P1 Conidia
}

\author{
Linda Gordon Hjeljord and Arne Tronsmo
}

Department of Chemistry and Biotechnology, Agricultural University of Norway, N-1432 Ås, Norway. Accepted for publication 9 July 2003.

\begin{abstract}
Hjeljord, L. G., and Tronsmo, A. 2003. Effect of germination initiation on competitive capacity of Trichoderma atroviride P1 conidia. Phytopathology 93:1593-1598.

Trichoderma biocontrol isolates are most effective as highly concentrated inocula. Their antagonism to other fungi may be a result of pregermination respiration. In a nutrient-rich medium, almost all Trichoderma atroviride $\mathrm{P} 1(\mathrm{P} 1)$ conidia initiated germination processes and increased respiration, even in dense suspensions. When $1 \times 10^{7} \mathrm{P} 1$ conidia/ml were coinoculated with $1 \times 10^{5}$ Botrytis cinerea conidia/ml, dissolved oxygen fell to $<1 \%$ within $2 \mathrm{~h}$ and the pathogen failed to germinate. More dilute

of $B$. cinerea were enhanced when P1 conidia were nutrient activated before inoculation. Pregermination respiration also affected competitive capacity of the antagonist on solid substrates, where respiratory $\mathrm{CO}_{2}$ stimulated germination rate and initial colony growth. These parameters were directly correlated with inoculum concentration $\left(R^{2} \geq 0.97, P<\right.$ 0.01). After initiating germination, Trichoderma conidia became more sensitive to desiccation and were killed by drying after only $2 \mathrm{~h}$ of incubation on a nutrient-rich substrate at $23^{\circ} \mathrm{C}$. These results indicate that nutrient-induced changes preceding germination in Trichoderma conidia can either enhance or decrease their biological control potential, depending on environmental conditions in the microhabitat.
\end{abstract} P1 suspensions consumed oxygen slowly enough to allow coinoculated $B$. cinerea to germinate. On nutrient-poor media, fewer P1 conidia initiated germination. Oxygen consumption by the inoculum and inhibition
Additional keywords: fungal ecophysiology, oxygen competition, Trichoderma harzianum.
The idea of applying large numbers of the saprophytic fungus Trichoderma spp. to plant surfaces in order to reduce disease caused by plant-pathogenic fungi dates back to at least the 1930s (29). Since then, many reports have confirmed the ability of Trichoderma spp. to suppress plant pathogens. Unfortunately, the disease control given by Trichoderma spp.-based commercial products is not yet consistent enough to ensure their widespread use by growers. In order to develop biological control programs reliable enough to be considered attractive alternatives to chemical fungicides, we need a better understanding of the competitive mechanisms involved in interactions between biocontrol fungi and their targets $(9,22,26)$.

The necessity of applying highly concentrated inocula of Trichoderma biocontrol agents to achieve effective disease control has been observed by many researchers, but not adequately explained. Foliar sprays containing $10^{7}$ to $10^{8}$ Trichoderma conidia/ml are considered necessary to suppress plant-pathogenic fungi such as Botrytis cinerea $(7,12,16)$. Thus, even small droplets of spray would contain thousands of conidia, seemingly many more than should be necessary for the antagonistic mechanisms generally thought to be involved in Trichoderma spp.-based biological control (e.g., antibiosis, mycoparasitism, and nutrient competition). Furthermore, these mechanisms may not fully explain the observed ability of Trichoderma spp. to inhibit faster-germinating pathogens (e.g., B. cinerea) when the antagonist conidia are inoculated together with, or just a few hours before, those of the pathogen $(15,23,30)$.

Previous investigations have shown that, when sufficiently concentrated, nutrient-activated Trichoderma conidia inhibit germination and blossom infection by $B$. cinerea to a greater degree than

Corresponding author: L. G. Hjeljord; E-mail address: linda.hjeljord@ikb.nlh.no

Publication no. P-2003-0930-02R

(c) 2003 The American Phytopathological Society do initially quiescent conidia (15). Inoculum concentration had a significant effect on the ability of the Trichoderma conidia to inhibit the pathogen in vitro. The present experiments were designed to examine more closely how inoculum concentration and nutrientinduced germination initiation affect the competitive capacity of Trichoderma conidia in aqueous media and on substrate surfaces.

\section{MATERIALS AND METHODS}

Fungal isolates. The antagonist strain used in these experiments was formerly known as T. harzianum strain P1 (ATCC 74058) (28), but it is now considered to belong to the species atroviride (19). The laboratory strain $B$. cinerea $\mathrm{E} 1$ originally was isolated from gray mold on strawberries in Norway. Conidia of both fungi were obtained from cultures grown on potato dextrose agar (PDA) (Difco Laboratories, Detroit, MI) for 2 weeks at 22 to $23^{\circ} \mathrm{C}$ under regular laboratory lighting. Conidia were harvested by flooding plates with sterile distilled water and rubbing the mycelia gently with a glass rod; suspensions of $B$. cinerea conidia were filtered through sterile cheesecloth before use. Conidia concentrations were determined by hemacytometer counts.

Germination assays. Germination in aqueous media was investigated by incubating $200 \mu \mathrm{l}$ of the conidial suspension at 21 to $23^{\circ} \mathrm{C}$ in wells of flat-bottom microtiter plates containing specific media and conidia concentrations. After $24 \mathrm{~h}$, germination was assessed visually using an invert microscope (Ernst Leitz Wetzlar $\mathrm{GmbH}$, Wetzlar, Germany) at $\times 400$ magnification. The first 100 conidia seen in each of two replicate wells per treatment were evaluated and counted as germinated when the germ tube length exceeded the diameter of the conidium. To assess germination and germ tube extension on agar, three replicate plates were inoculated with drops of conidia suspensions and incubated as indicated. Measurements were made on individual germ tubes (average length of 20 hyphae per plate on three replicate plates) until these extended beyond the perimeter of the inoculum drop, after which radial growth of the colony was measured macroscopically. 
Nutrient activation. Nutrient activation of $T$. atroviride $\mathrm{P} 1$ conidia was performed as described previously (15). Briefly, conidia were incubated in $2.4 \%$ (wt/vol) potato dextrose broth (PDB) at 21 to $23^{\circ} \mathrm{C}$ on a reciprocal shaker at $150 \mathrm{rpm}$ for 6 to $7 \mathrm{~h}$, pelleted and washed twice in sterile distilled water by centrifugation, resuspended in sterile distilled water at a concentration of approximately $10^{9} \mathrm{conidia} / \mathrm{ml}$, and stored at $4^{\circ} \mathrm{C}$ until used. During activation, the diameter of $\geq 90 \%$ of the conidia increased from 3 to $3.5 \mu \mathrm{m}$ (quiescent conidia) to 6 to $7.5 \mu \mathrm{m}$ (swollen conidia). No germ tubes were produced during the incubation period.

Desiccation experiments. The effect of substrate nutrient concentration and inoculum concentration on germination initiation in T. atroviride P1 inocula was investigated through desiccation experiments. Conidia were inoculated in various concentrations of nutrient media in microtiterplate wells as described above. After various periods of incubation, $10-\mu$ drops were removed from the nutrient media and inoculated onto three replicate polysulphone membrane filters (9 $\mathrm{cm}$ in diameter) (Gelman Sciences, Ann Arbor, MI) placed in sterile uncovered petri dishes. These were left to dry overnight in a laminar flow cabinet. After drying, the membranes were placed on fresh PDA plates and incubated at room temperature $\left(21\right.$ to $\left.23^{\circ} \mathrm{C}\right)$. After a given time (e.g., $36 \mathrm{~h}$ ), the radius of the mycelium growing from the dried inoculum was measured and compared with mycelia produced after the same incubation time by undried inocula of known conidia concentrations. This experiment was repeated three times. In other experiments, various concentrations of conidia were incubated in $10-\mu l$ drops on membrane filters placed on agar plates varying in nutrient concentration. Two replicate filters were inoculated at each nutrient level. After a specified incubation period, the inoculated membranes were removed, dried, and replaced on fresh agar plates as described above. This experiment was repeated twice. The linear correlation between (undried) inoculum concentration and subsequent colony radius permitted estimation of the proportion of viable conidia remaining in the dried inoculum (described below). When the number of germinating conidia in the inoculum was small enough to be counted, this was done to confirm the estimation, because the correlation between colony radius and inoculum concentration was best for concentrations of $\geq 10^{5}$ conidia $\mathrm{ml}^{-1}$.

Oxygen consumption. Oxygen depletion by $T$. atroviride P1 conidia in liquid media was monitored using oxygen electrodes (7-ml perspex electrode and Digital model 10 controller; Rank Brothers, Cambridge, England). All tests were performed at 21 to $23^{\circ} \mathrm{C}$. A mixture of nutrient medium and conidia (concentrations of each as specified) was oxygenated by shaking vigorously ("100\% air saturation") and $4 \mathrm{ml}$ was placed in the oxygen monitoring chamber, which then was closed. The conidia suspension

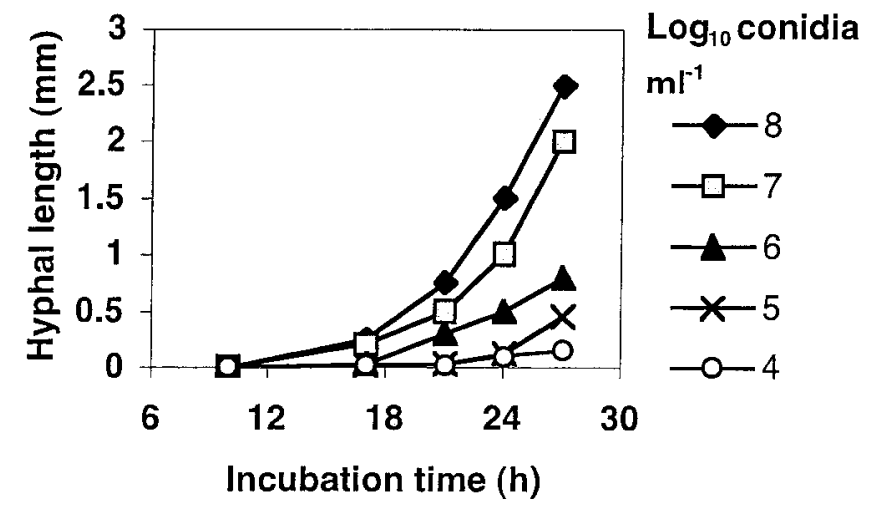

Fig. 1. Effect of inoculum concentration on radial extension in young Trichoderma atroviride P1 colonies. Conidia suspensions were inoculated in 5 - $\mu \mathrm{l}$ drops on potato dextrose agar plates and incubated at $23^{\circ} \mathrm{C}$. Data points are means of measurements on three replicate plates in a single experiment representative of repeated experiments; standard deviation bars are below the figure resolution. was stirred continuously while oxygen content was monitored and expressed as percentage of air saturation. Oxygen was considered to be depleted when no further decrease was detectable, which always occurred at less than $1 \%$ air saturation. Oxygen consumption by the various inocula was monitored in separate experiments at least twice, using different electrodes. The results presented are representative of repeated experiments.

$\mathrm{CO}_{2}$ removal. Effect of $\mathrm{CO}_{2}$ on $T$. atroviride $\mathrm{P} 1$ germination and colony growth was examined by removal of $\mathrm{CO}_{2}$ from the air over cultures on PDA. Two milliliters of $10 \%$ (wt/vol) $\mathrm{KOH}$ or sterile distilled water (control) was added to 7-cm diameter disks of Whatman No. 1 filter paper placed in the lids of sterile petri dishes (24). PDA plates were inoculated with $10 \mu \mathrm{l}$ of $T$. atroviride conidia suspensions in water and inverted over the lids with filter paper. The plates were sealed with parafilm and incubated at $23^{\circ} \mathrm{C}$ for $20 \mathrm{~h}$, after which germination was assessed microscopically. In a second experiment, the filter paper size was reduced, so that only part of the petri dish lid was covered, thus allowing nondestructive assessment of germination and colony growth for 3 days. Each experiment was done using four replicate plates per treatment and was repeated once.

Experimental design and statistics. The germination tests in microtiter plates included two replicate wells per treatment, set up as a factorial, complete block design, with columns or rows of wells as blocks. Wells were assessed in randomized order. Radial extension was measured on three replicate plates per treatment (inoculum or PDB concentration). The plates were incubated in plastic bags, each containing one replicate of each treatment arranged in randomized order. For most assays reported, data from a single representative experiment were used to illustrate results from multiple similar experiments.

Inoculum and substrate concentration data were $\log _{10}$ transformed before analysis. Regression analysis was used to investigate effects of inoculum and substrate concentrations on germination and radial extension. The combined effects of inoculum and substrate concentrations on germination were analyzed by twoway analysis of variance. Statistical analyses were performed using the statistics software packages Excel Data Analysis Toolpak (Microsoft Corp., Redmond, WA) and StatView 5.0.1 (SAS Institute, Cary, NC).

\section{RESULTS}

Effect of inoculum concentration on germination and growth. The number of $T$. atroviride $\mathrm{P} 1$ conidia in the inoculum affected the size and growth rate of the young colony on agar plates (Figs. 1 and 2). The average extension rate during the first

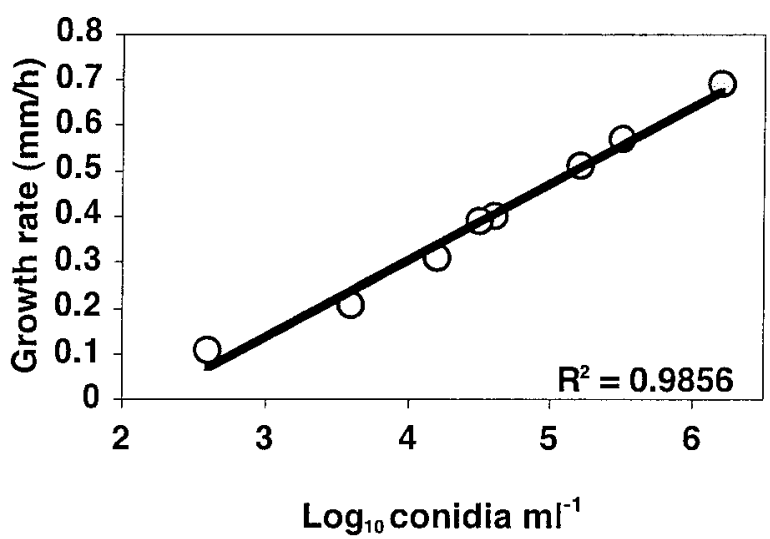

Fig. 2. Inoculum concentration significantly affected growth rate of Trichoderma atroviride P1 germ tubes during the first $18 \mathrm{~h}$ following germination on potato dextrose agar $(P<0.001)$. Data points represent the mean of two replicates and are taken from two independent experiments. 
$72 \mathrm{~h}$ after inoculation was $7.3 \pm 0.1 \mu \mathrm{m} \min ^{-1}$ (standard deviation) for conidia germinating in an inoculum of $10^{5}$ conidia $\mathrm{ml}^{-1}$, whereas the rate was $11.1 \pm 0.4 \mu \mathrm{m} \mathrm{min}{ }^{-1}$ from an inoculum of $10^{7}$ conidia $\mathrm{ml}^{-1}$. The radius of a $T$. atroviride $\mathrm{P} 1$ colony growing from a concentrated inoculum was greater after a given incubation period than that of a colony originating from fewer conidia; therefore, it was possible to roughly estimate the number of conidia germinating in an unknown inoculum by comparison with colonies of the same age produced by inocula of known concentration. This technique was used to examine the effect on $T$. atroviride P1 inocula of being dried before germinating (described below).

The increased growth rate from concentrated inocula may be the result of a more rapid germination rate. Germination was practically complete $17 \mathrm{~h}$ after inoculation on a plate inoculated with $2 \times 10^{8}$ conidia $\mathrm{ml}^{-1}$, but was just starting in an inoculum of $10^{4}$ conidia $\mathrm{ml}^{-1}$ (conidia in air; Fig. 3). Germination in all but the most concentrated inoculum was slowed when $\mathrm{CO}_{2}$ was removed from the culture air through absorption by $\mathrm{KOH}$ (Fig. 3).

Although the above results demonstrated self-stimulation of germination in air, conidia in concentrated aqueous suspensions showed self-inhibition. In a previous study, it was observed that Trichoderma conidia settle out of suspension in still culture and accumulate at the bottom of inoculum drops on plant surfaces or on the bottom of microtiterplate wells. Even under otherwise conducive conditions, germination percent in such accumulations decreases with increasing conidia concentration in inocula containing more than $10^{6}$ conidia $\mathrm{ml}^{-1}$ (e.g., to approximately $10 \%$ in inocula of $10^{8}$ conidia $\mathrm{ml}^{-1}$ ) (15). To determine whether such ungerminated conidia were inhibited before or after initiating germination, inocula of varying densities were incubated on membrane filters on PDA for various periods and then dried. When the dried filters subsequently were placed on PDA, the radii of colonies growing from the dried inocula were compared with those originating from undried inocula of known conidia concentrations to estimate the percentage of germinable conidia remaining in the dried inocula (Fig. 1). In inocula dried without incubation on PDA, practically all conidia survived the drying procedure (Fig. 4). However, incubation in the nutrient-rich medium for only $2 \mathrm{~h}$ induced cellular changes which rendered conidia sensitive to drying. More than $90 \%$ of the conidia were killed by desiccation following contact with nutrients, even in the most concentrated inocula (Fig. 4).

Effect of substrate nutrients on germination initiation. In $T$. atroviride conidia, germination percentage is directly correlated with the level of available nutrients, even in relatively dilute conidia suspensions (Fig. 5). This appears to reflect physiological characteristics of the conidia population rather than nutrient com-

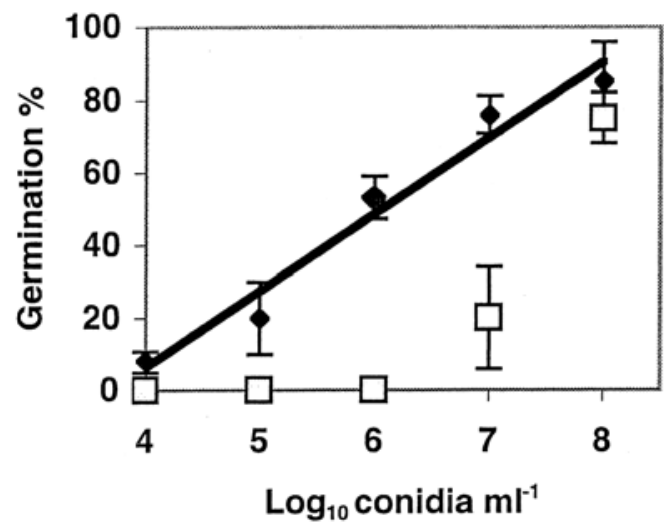

Conidia germinating over:

\section{- Air} $\square \mathrm{KOH}$

Fig. 3. The percentage of Trichoderma atroviride $\mathrm{P} 1$ which had completed germination $17 \mathrm{~h}$ after inoculation was positively affected by inoculum concentration when conidia were incubated in air $\left(R^{2}=0.967, P<0.001\right)$. When $\mathrm{CO}_{2}$ was removed by $\mathrm{KOH}$, the effect of inoculum concentration was reduced $\left(R^{2}=0.685, P=0.08\right.$; trendline not shown). Data points are means ( \pm standard deviation) of four replicate plates. petition, because the percentage of germination in the various inocula was significantly $(P<0.001)$ affected by PDB concentration, but not by conidia concentration $(P>0.1)$. Desiccation experiments demonstrated that the ungerminated conidia had failed to initiate germination on nutrient-poor substrates, because these survived drying and germinated when they were placed later on a nutrient-rich medium (Fig. 6). A suspension incubated overnight in distilled water was not affected by the drying procedure (data not shown).

Effect of germination initiation on antagonism. $B$. cinerea and $T$. atroviride conidia were coinoculated in microtiter-plate wells containing various concentrations of PDB, and germination of the pathogen conidia was assessed after $24 \mathrm{~h}$. The effect of preliminary germination initiation on antagonistic potential of $T$. atroviride conidia was tested by incubating conidia in PDB for $7 \mathrm{~h}$ before they were washed and coinoculated with $B$. cinerea in fresh media. Nutrient-activated but ungerminated conidia suppressed

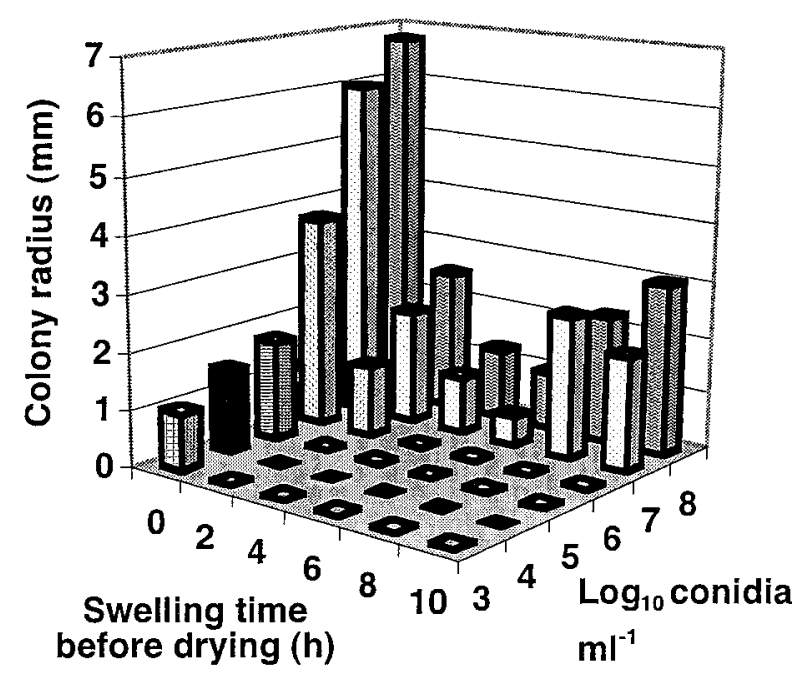

Fig. 4. Contact with nutrients induces germination initiation in Trichoderma atroviride $\mathrm{P} 1$ conidia, which renders them sensitive to desiccation. Conidia were incubated in potato dextrose broth at $23^{\circ} \mathrm{C}$ for various periods before being inoculated onto filter membranes and dried overnight. The membranes then were placed on potato dextrose agar for resumption of growth, and the resulting mycelium was measured at $36 \mathrm{~h}$. Data points are means of three replicate treatments in a single experiment representative of repeated experiments; average standard error $=0.25 \mathrm{~mm}$.

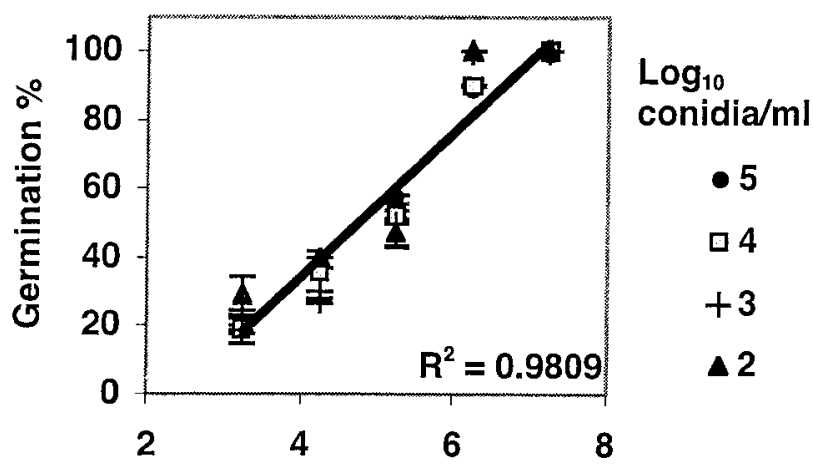

PDB concentration $\left(\log _{10} \mu \mathrm{g} / \mathrm{l}\right)$

Fig. 5. Substrate nutrient level affected germination percent of Trichoderma atroviride, even in dilute conidia suspensions. Inocula were incubated for $24 \mathrm{~h}$ at $23^{\circ} \mathrm{C}$ in potato dextrose broth dilutions. The trendline shown is for $1 \times 10^{5}$ conidia per $\mathrm{ml}(P<0.001) . R^{2}$ values for the other conidia concentrations were $0.8721(P=0.02), 0.9115(P=0.01)$, and $0.9681(P=0.002)$ for $10^{2}, 10^{3}$, and $10^{4}$ conidia/ml, respectively. Data points are means ( \pm standard deviation) of two replicates from a single experiment representative of repeated experiments. 
germination of $B$. cinerea more consistently and to a greater degree than fresh conidia at all nutrient levels tested (Fig. 7). Initially quiescent $T$. atroviride conidia varied in their effect on $B$. cinerea germination (mean standard error between experiments on the substrates shown $=15.0 \%$ ) When nutrient activated before inoculation, a concentration of $1 \times 10^{7} \mathrm{~T}$. atroviride conidia $\mathrm{ml}^{-1}$ reduced $B$. cinerea germination by $\geq 97 \%$ (mean standard error $=$ $5.7 \%$ ) on all media tested. Substrate nutrient levels did not significantly affect the inhibition shown by either fresh or activated conidia $(P>0.5)$.

Oxygen consumption by ungerminated conidia. Conidial respiration following initiation of germination was monitored by measuring dissolved oxygen levels in inoculated media. The total conidia concentration in the medium affected the speed of oxygen depletion (Fig. 8). When PDB (24 mg ml${ }^{-1}$ ) was inoculated with $1 \times 10^{5} \mathrm{~B}$. cinerea conidia $\mathrm{ml}^{-1}$, an oxygen deficit in the medium occurred approximately $5 \mathrm{~h}$ after inoculation. When the suspen-

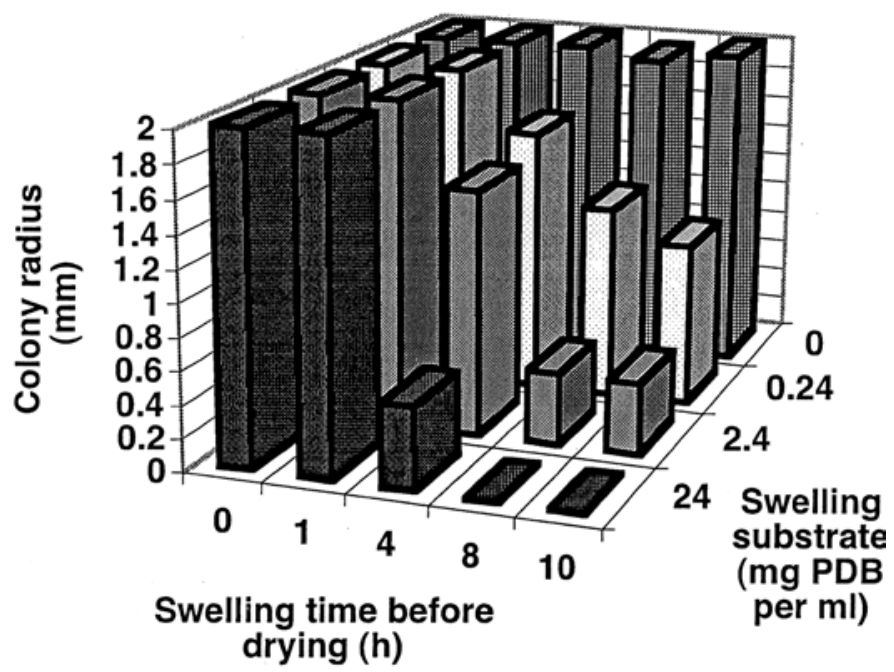

Fig. 6. Substrate composition affected the number of conidia in a Trichoderma atroviride inoculum which initiated germination and became sensitive to desiccation. Drops $(10 \mu \mathrm{l})$ containing $1 \times 10^{5}$ conidia $\mathrm{ml}^{-1}$ were placed on membrane filters on agar plates differing in nutrient content. At various times during the incubation, filters were removed and dried, then placed on potato dextrose agar $\left(24 \mathrm{mg} \mathrm{ml}^{-1}\right)$ for regrowth. Radii of surviving colonies were measured after $36 \mathrm{~h}$. Data are means of two replicate plates in a single experiment representative of repeated experiments.

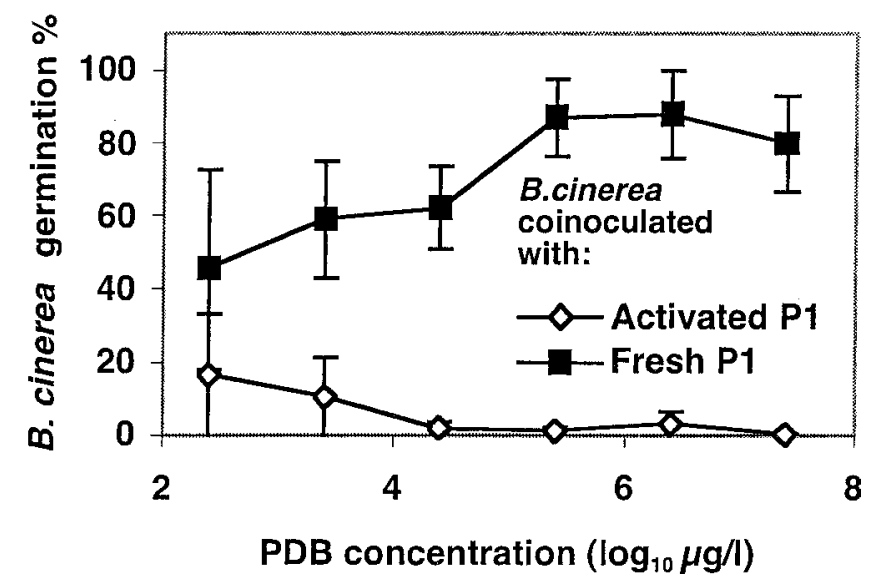

Fig. 7. Effect of preliminary nutrient activation on competitive ability of Trichoderma atroviride conidia. Botrytis cinerea conidia $\left(1 \times 10^{5}\right.$ conidia ml $\left.^{-1}\right)$ were coinoculated with nutrient-activated or fresh $T$. atroviride $\mathrm{P} 1$ conidia $\left(1 \times 10^{7} \mathrm{ml}^{-1}\right)$. Germination of $B$. cinerea was assessed after $24 \mathrm{~h}$ and is expressed as percentage of germination in axenic $B$. cinerea culture on the various substrates. Bars are means ( \pm standard error) of three separate experiments. sion of $B$. cinerea was coinoculated with $1 \times 10^{5} T$. atroviride $\mathrm{P} 1$ conidia $\mathrm{ml}^{-1}$, oxygen levels remained above $1 \%$ for $\geq 4 \mathrm{~h}$. However, when the $B$. cinerea conidia were coinoculated with $1 \times$ $10^{7}$ T. atroviride $\mathrm{P} 1$ conidia $\mathrm{ml}^{-1}$, dissolved oxygen fell below $1 \%$ saturation within $\approx 2 \mathrm{~h}$ (Fig. 8). After $7 \mathrm{~h}$, the chambers were opened to determine germination: $43 \pm 0.6 \%$ of the $B$. cinerea conidia had germinated in the chamber without T. atroviride $\mathrm{P} 1$ conidia, and $41 \pm 1.2 \%$ had germinated in the presence of $1 \times$ $10^{5}$ antagonist conidia $\mathrm{ml}^{-1}$, but none had germinated in the presence of $1 \times 10^{7} T$. atroviride $\mathrm{P} 1$ conidia $\mathrm{ml}^{-1}$. The latter suspension was oxygenated, and within $2 \mathrm{~h}, 66 \pm 4.1 \%$ of the $B$. cinerea conidia had produced germ tubes (data are means and standard deviations of three replicates). B. cinerea germination after $24 \mathrm{~h}$ in microtiterplate wells was reduced, but not prevented (Fig. 7), by $1 \times 10^{7}$ fresh P1 conidia $\mathrm{ml}^{-1}$ in PDB at $24 \mathrm{mg} \mathrm{ml}^{-1}$. These results could indicate that germination was slowed by low oxygen concentrations, but also may reflect the variability between experiments at a concentration of $1 \times 10^{7}$ fresh $T$. atroviride conidia $\mathrm{ml}^{-1}$, which is the lowest effective concentration. The mean $B$. cinerea germination in the presence of $1 \times 10^{7}$ fresh $T$. atroviride conidia at this nutrient concentration was $80 \pm 23 \%$ (Fig. 7).

Effect of nutrient activation on oxygen depletion rate of $\boldsymbol{T}$. atroviride $\mathbf{P 1}$ conidia. There was an inverse correlation between the minutes elapsed before an oxygen deficit occurred in the medium $(D)$ and the $\log _{10}$ concentration of $T$. atroviride $\mathrm{P} 1$ conidia $(C): D=-5.4(C)+42.5, R^{2}=0.95, P=0.001$ (Fig. 9). When the Trichoderma conidia were activated before inoculation, the inoculum concentration necessary to deplete the medium of oxygen by a given time was reduced by approximately an order of magnitude: $D=-3.1(C)+22.9, R^{2}=0.93, P=0.002$.

It is not known how long nutrient-activated conidia retain a higher respiratory rate during storage. Activated conidia $(2 \times$ $10^{7} \mathrm{ml}^{-1}$ ) which had been stored in water at $4^{\circ} \mathrm{C}$ for 10 days were found to deplete oxygen in a wide range of nutrient media significantly faster than the same concentration of initially-quiescent conidia ( $t$ test, $P<0.05$ ), although a sample of activated conidia stored for 14 months was not significantly different from fresh conidia with regard to oxygen depletion rate (data not shown). The average germination percentage in eight batches of activated conidia stored for up to 18 months was not significantly different

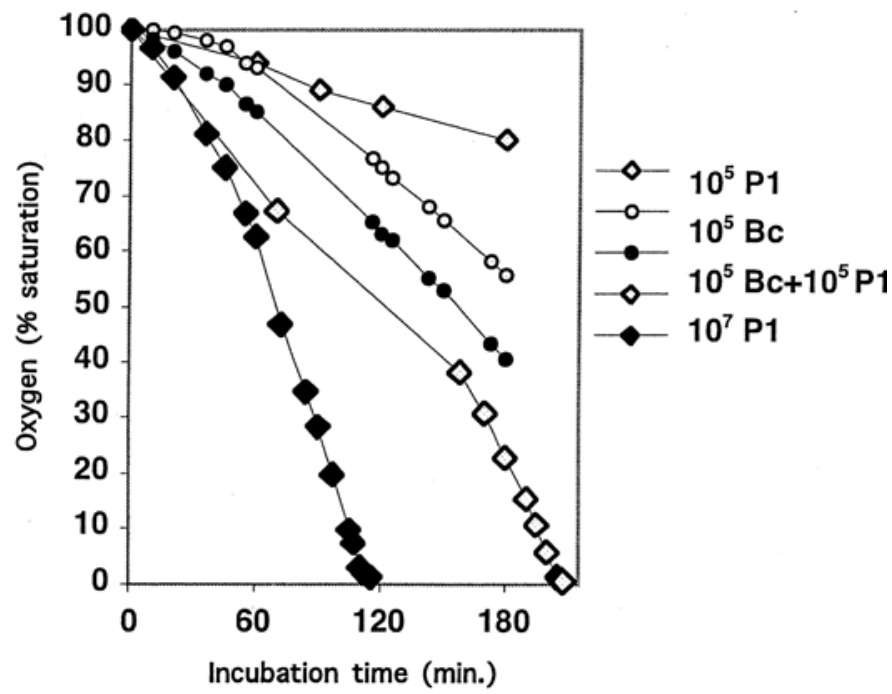

Fig. 8. Oxygen depletion from a medium coinoculated with Botrytis cinerea and Trichoderma atroviride P1 conidia was the result of respiration by both fungi and was affected by conidia concentration. Conidia were incubated in potato dextrose broth at $24 \mathrm{mg} \mathrm{ml}^{-1}$ and $23^{\circ} \mathrm{C}$. Dissolved oxygen in the medium was monitored by oxygen electrodes and expressed as percent air saturation. Data shown are from a single experiment representative of repeated experiments. 
from that of fresh conidia on the nutrient media shown in Figure 5 and remained significantly higher $(t$ test, $P=0.016)$ than that of fresh conidia in distilled water $(25 \pm 7 \%$ germination in activated conidia, $5 \pm 2 \%$ in fresh conidia [mean $\pm \mathrm{SE}]$ ).

\section{DISCUSSION}

The necessity of applying highly concentrated inocula of Trichoderma spp. to achieve satisfactory biological control is well established, despite the apparent paradox that highly concentrated conidia suspensions produce not only superior disease control but also considerable self-inhibition of germination $(13,15)$. However, our results show that evaluating antagonistic potential of biocontrol isolates on the basis of germination percentage overlooks an important point: whether or not they actually germinate, the cellular changes conidia undergo in preparation for germination may enhance or inhibit their further activity, depending on the specific microenvironment.

One of the first changes induced in quiescent Trichoderma conidia by a germination-conducive substrate is altered wall permeability $(1,25)$. Due to the fairly synchronous initiation of germination in even quite concentrated suspensions, most or all of an inoculum quickly becomes sensitive to desiccation on a nutrientrich substrate. This period of susceptibility lasts until $\approx 9$ to $11 \mathrm{~h}$ after germination (at 20 to $25^{\circ} \mathrm{C}$ ), at which time the germ tubes become competent to respond to insufficient water availability with microconidiation (31; L. G. Hjeljord, unpublished observation). In practical terms, this means that most or all of the inoculum may be killed by drying within $24 \mathrm{~h}$ of application to a nutrientrich substrate if temperatures are conducive to rapid germination, while the period of sensitivity will be longer at lower temperatures.

Another result of germination initiation that has consequences for biological control is an immediate increase in respiration and oxygen consumption by the antagonist conidia upon contact with nutrients $(4,11)$. B. cinerea is more sensitive to low oxygen levels in wet microsites than are Trichoderma spp. $(5,10,20)$; therefore, it appears that respiration by the antagonist conidia could result in an oxygen deficit sufficient to prevent $B$. cinerea germination in flower parts. Although such antagonism could be effective only under conditions allowing persistence of water in nutrient-rich microhabitats (e.g., moist flower parts containing nectar and pollen exudates), such conditions are just those supporting infection by opportunistic pathogens such as B. cinerea $(2,8,17)$.

In the experiments reported, it was necessary to stir the conidia suspensions to accurately measure dissolved oxygen. When sprayed onto a surface, conidia settle out of suspension into accumulations considerably more concentrated than in stirred suspensions. Due to the correlation between conidia concentration and oxygen depletion rate, the results presented likely underestimate the oxygen depletion rate of concentrated antagonist inocula accumulating in natural microsites. Although the water layer in such sites may be thin, it has been noted that even films of water surrounding respiring hyphae can become depleted of oxygen, due to the low solubility of oxygen in water (6).

There are other antagonistic mechanisms that suppress germination of $B$. cinerea, but none seem able to explain the inhibition seen in our experiments. For example, many microorganisms can inhibit B. cinerea germination through nutrient competition (3). Although competition for nutrients may have been involved, inhibition of $B$. cinerea germination in the presence of concentrated Trichoderma inocula was not significantly affected by substrate nutrient levels in our experiments. Another possible antagonistic mechanism is production of inhibitory enzymes or metabolites. Although Trichoderma spp. possess preformed conidia-bound enzymes which are active in preliminary substrate degradation before germination, these are not secreted into the medium (18). All reports of inhibitory extracellular enzyme production by Tricho- derma spp. have, to our knowledge, been based on culture filtrates from mycelia, not conidia. We previously have reported that, when inhibited B. cinerea and Trichoderma conidia are removed from densely inoculated media, the culture filtrate supports full germination of more dilute inocula (15), which indicates that secreted enzymes or metabolites are not the cause of the inhibition.

On a microhabitat in which oxygen was not limiting (agar plates), there was a direct correlation between the number of $T$. atroviride conidia in the inoculum and their germination and hyphal extension rates. $\mathrm{CO}_{2}$ amounts slightly exceeding those in air are known to stimulate germination and growth of many fungi which can fix $\mathrm{CO}_{2}$, including Trichoderma spp. $(5,24,27)$. If $\mathrm{CO}_{2}$ levels in air are insufficient to support a maximal rate of biosynthesis during spore germination in concentrated inocula, locally elevated $\mathrm{CO}_{2}$ levels derived from respiration of adjacent cells would be self-stimulatory for the population. This assumption was supported by the results showing that dense accumulations of conidia were less inhibited than dispersed conidia when $\mathrm{CO}_{2}$ was removed from the air over the cultures.

Germination in a Trichoderma inoculum is affected by the nutrient status of the substrate; therefore, a percentage of the inoculum may fail to initiate germination if applied to a previously colonized microsite (e.g., senescing flower parts). The importance of applying antagonists to newly opened flowers is well known. Nutrient dependence of commercially formulated Trichoderma isolates can be even more pronounced than that seen in fresh conidia (14). This could be another reason why concentrated inocula have better effect in biological control (i.e., germination initiation in $50 \%$ of an inoculum of $10^{7}$ conidia $\mathrm{ml}^{-1}$ would produce more activity than $50 \%$ of $10^{5}$ conidia $\mathrm{ml}^{-1}$ ).

Deliberate nutrient activation of Trichoderma conidia prior to application appears to have the potential to improve the efficacy of Trichoderma spp.-based biological control programs, particularly under environmental conditions leading to reduced germination in the inoculum. It remains to be determined how long activated conidia retain enhanced competitive capacity during storage. Although an increased rate of endogenous respiration has been reported to decrease spore viability (21), activated conidia are highly concentrated during storage and their respiration rate may be lowered by anaerobiosis. Preliminary results indicate that activated $T$. atroviride $\mathrm{P} 1$ conidia can be stored for at least a year

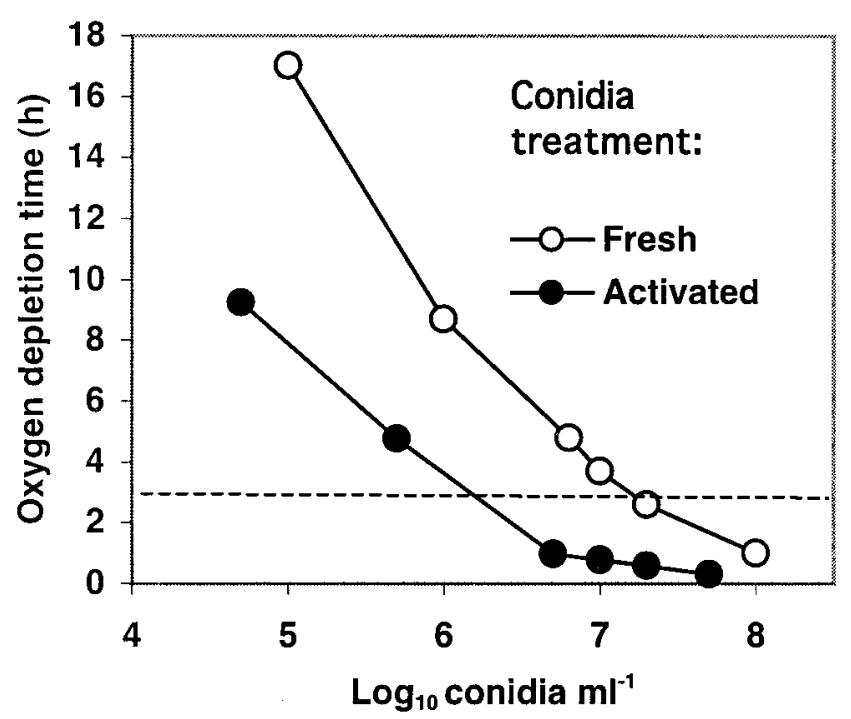

Fig. 9. Oxygen consumption by Trichoderma atroviride $\mathrm{P} 1$ conidia was affected by inoculum concentration and preliminary nutrient activation. The dashed line indicates the approximate time at which Botrytis cinerea conidia initiate germination under these conditions, to illustrate the importance of antagonist conidia concentration in creating an oxygen deficit quickly enough to inhibit the pathogen. Each data point is the mean of two experiments; average standard deviation $= \pm 0.15 \mathrm{~h}$. 
without losing viability. The effects of storage conditions on respiration and germinability of activated conidia are currently under investigation.

In summary, the results presented indicate that the often-observed importance of using highly concentrated inocula of Trichoderma for biological control could be due to nutrient-dependent germination initiation. Even in concentrated inocula, most conidia initiate germination simultaneously in nutrient-rich microsites. The consequent increase in respiration can create an oxygen deficit in wet microsites which can prevent germination not only of the antagonist but also of other fungi. Thus, competition for oxygen could be an effective biological control mechanism in wet, nutrient-rich microsites. In contrast, respiration in concentrated Trichoderma inocula is self-stimulatory in aerobic microhabitats, and the enhanced growth rate could improve the capacity of the antagonist to compete for nutrients (e.g., through production of inhibitory enzymes and metabolites). However, we have shown that, on nutrient-poor substrates, a proportion of the conidia population failed to initiate germination, even in dilute inocula. On such sites, application of concentrated inocula would result in germination of greater numbers of antagonist conidia. The ability of Trichoderma conidia to compete for oxygen and nutrients is improved if they initiate germination before arrival of the pathogen. This can be achieved by expedient timing of application to nutrient-rich microsites (e.g., newly opened flowers) or by preliminary treatment of the inoculum to activate germination processes before application. A negative consequence of germination initiation is increased sensitivity to desiccation. Although such severe drying as used in vitro would be unlikely to occur on transpiring leaves or flowers, this factor could be relevant if conidia are applied to hard fruit surfaces (e.g., to prevent wound infection). Consideration of these ecophysiological factors may improve prediction of the results of application of Trichoderma conidia to aboveground plant surfaces under varying environmental conditions and thus may ultimately improve efficacy of Trichoderma spp.-based biological control programs.

\section{ACKNOWLEDGMENTS}

This research was supported financially by projects funded by the Norwegian Industrial and Regional Development Fund (SND) and the Research Council of Norway, including the Strategic Institute Program no. 147029/140, "Use of beneficial fungi to control weeds, insect pests and plant pathogenic fungi". We thank S. Olsson, E. Koch, and R. A. Olsen for their valuable discussions of this research.

\section{LITERATURE CITED}

1. Barnes, M., and Parker, M. S. 1966. The increase in size of mould spores during germination. Trans. Br. Mycol. Soc. 49:487-494.

2. Blakeman, J. P. 1980. Behaviour of conidia on aerial plant surfaces. Pages 115-151 in: The Biology of Botrytis. J. R. Coley-Smith, K. Verhoeff, and W. R. Jarvis, eds. Academic Press, London.

3. Brodie, I. D. S., and Blakeman, J. P. 1976. Competition for exogenous substrates in vitro by leaf surface micro-organisms and germination of conidia of Botrytis cinerea. Physiol. Plant Pathol. 9:227-239.

4. Cochrane, V. W. 1966. Respiration and spore germination. Pages 201213 in: The Fungus Spore. M. F. Madelin, ed. Butterworths, London.

5. Danielson, R. M., and Davey, C. B. 1973. Non nutritional factors affecting the growth of Trichoderma in culture. Soil Biol. Biochem. 5:495-504.

6. Deacon, J. W. 1997. Modern Mycology, 3rd ed. Blackwell Science, Cambridge.

7. Dubos, B. 1987. Fungal antagonism in aerial agrobiocenoses. Pages 107135 in: Innovative Approaches to Plant Disease Control. I. Chet, ed. John Wiley \& Sons, New York.
8. Fourie, J. F., and Holz, G. 1998. Effects of fruit and pollen exudates on growth of Botrytis cinerea and infection of plum and nectarine fruit. Plant Dis. 82:165-170.

9. Fravel, D. 1999. Hurdles and bottlenecks on the road to biocontrol of plant pathogens. Australas. Plant Pathol. 28:53-56.

10. Gochenaur, S. E. 1981. Response of soil fungal communities to disturbance. Pages 459-479 in: The Fungal Community. D. T. Wicklow and G. C. Carroll, eds. Marcel Dekker, New York.

11. Gottlieb, D. 1978. The Germination of Fungal Spores. Patterns of Progress. J. G. Cook, ed. Meadowfield Press Ltd., Durham, England.

12. Gullino, M. L. 1992. Control of Botrytis rot of grapes and vegetables with Trichoderma spp. Pages 125-132 in: Biological Control of Plant Diseases. E. C. Tjamos, G. C. Papavizas, and R. J. Cook, eds. Plenum Press, New York.

13. Harman, G. E., and Björkman, T. 1998. Potential and existing uses of Trichoderma and Gliocladium for plant disease control and plant growth enhancement. Pages 229-265 in: Trichoderma \& Gliocladium: Enzymes, Biological Control and Commercial Applications. G. E. Harman and C. P. Kubicek, eds. Taylor \& Francis, Ltd., London.

14. Hjeljord, L. G., Stensvand, A., and Tronsmo, A. 2000. Effect of temperature and nutrient stress on the capacity of commercial Trichoderma products to control Botrytis cinerea and Mucor piriformis in greenhouse strawberries. Biol. Control 19:149-160.

15. Hjeljord, L. G., Stensvand, A., and Tronsmo, A. 2001. Antagonism of nutrient-activated conidia of Trichoderma harzianum (atroviride) P1 against Botrytis cinerea. Phytopathology 91:1172-1180.

16. Hong, C. X., Michailides, T. J., and Holtz, B. A. 1998. Effects of wounding, inoculum density, and biological control agents on postharvest brown rot of stone fruits. Plant Dis. 82:1210-1216.

17. Jarvis, W. R. 1977. Botryotinia and Botrytis species: Taxonomy, physiology and pathogenicity. Can. Dep. Agric. Monogr. No. 15.

18. Kubicek, C. P., Mühlbauer, G., Klotz, M., John, E., and Kubicek-Pranz, E. M. 1988. Properties of a conidial-bound cellulase enzyme system from Trichoderma reesei. J. Gen. Microbiol. 134:1215-1222.

19. Kullnig, C. M., Krupica, T., Woo, S. L., Mach, R. L., Rey, M., Benítez, T., Lorito, M., and Kubicek, C. P. 2001. Confusion abounds over identities of Trichoderma biocontrol isolates. Mycol. Res. 105:770-772.

20. Macauley, B. J., and Griffin, D. M. 1969. Effects of carbon dioxide and oxygen on the activity of some soil fungi. Trans. Br. Mycol. Soc. 53:5362 .

21. Mandels, G. R. 1963. Endogenous respiration of fungus spores in relation to dormancy and germination. Ann. N.Y. Acad. Sci. 102:724739.

22. Nelson, E. B. 1991. Current limits to biological control of fungal phytopathogens. Pages 327-355 in: Handbook of Applied Mycology: Soil and Plants. D. K. Arora, B. Rai, K. G. Mukerji, and G. R. Knudsen, eds. Marcel Dekker, New York.

23. O’Neill, T. M., Niv, A., Elad, Y., and Shtienberg, D. 1996. Biological control of Botrytis cinerea on tomato stem wounds with Trichoderma harzianum. Eur. J. Plant Pathol. 102:635-643.

24. Robinson, P. M., and Thompson, L. A. A. 1982. Volatile promoter of germination and hyphal extension produced by Geotrichum candidum. Trans. Br. Mycol. Soc. 78:353-383.

25. Rosen, D., Edelman, M., Galun, E., and Danon, D. 1974. Biogenesis of mitochondria in Trichoderma viride: Structural changes in mitochondria and other spore constituents during conidium maturation and germination. J. Gen. Microbiol. 83:31-49.

26. Stewart, A. 2001. Commercial biocontrol-reality or fantasy? Australas. Plant Pathol. 30:127-131.

27. Tabak, H. H., and Cooke, W. B. 1968. The effects of gaseous environments on the growth and metabolism of fungi. Bot. Rev. 34:126-252.

28. Tronsmo, A. 1989. Effect of fungicides and insecticides on growth of Botrytis cinerea, Trichoderma viride and T. harzianum. Norw. J. Agric. Sci. 3:151-156.

29. Weindling, R. 1932. Trichoderma lignorum as a parasite of other soil fungi. Phytopathology 22:837-845.

30. Wood, R. K. S. 1951. The control of diseases of lettuce by the use of antagonistic organisms. 1. The control of Botrytis cinerea Pers. Ann. Appl. Biol. 38:203-216.

31. Zuber, J., and Turian, G. 1981. Induction of premature phialoconidiogenesis on germinated conidia of Trichoderma harzianum. Trans. Br. Mycol. Soc. 76:433-440. 